\title{
BMJ Open Technology-based counselling in dementia (TeCoDem): study protocol of a mixed-methods systematic review with qualitative comparative analysis and meta-analysis
}

\author{
Julian Hirt (D) , ${ }^{1,2,3}$ Gero Langer, ${ }^{1}$ Fabian Wilde, ${ }^{1}$ Dorothee Bauernschmidt, ${ }^{1}$ \\ Gabriele Meyer, ${ }^{1}$ Anja Bieber (i) ${ }^{1}$
}

To cite: Hirt J, Langer G, Wilde F, et al. Technologybased counselling in dementia (TeCoDem): study protocol of a mixed-methods systematic review with qualitative comparative analysis and meta-analysis. BMJ Open 2021;11:e054157. doi:10.1136/ bmjopen-2021-054157

- Prepublication history and additional supplemental material for this paper are available online. To view these files, please visit the journal online (http://dx.doi.org/10.1136/ bmjopen-2021-054157)

Received 05 June 2021 Accepted 15 November 2021

D) Check for updates

(c) Author(s) (or their employer(s)) 2021. Re-use permitted under CC BY-NC. No commercial re-use. See rights and permissions. Published by BMJ.

For numbered affiliations see end of article.

\section{Correspondence to}

Dr Anja Bieber;

anja.bieber@medizin.uni-halle. de

\section{ABSTRACT}

Introduction Information technology can enhance timely and individual support for people with dementia and informal carers. There is some evidence that people with dementia and informal carers would benefit from technology-based counselling. However, it remains unclear which features of those interventions relate to beneficial outcomes and which aspects are necessary for a successful implementation. Therefore, the primary objectives are: (1) to identify conditions of successful implementation of technology-based counselling interventions in dementia and (2) to investigate the effectiveness of those interventions.

Methods and analysis We will conduct a mixedmethods systematic review. The first primary objective requires evidence from various study designs addressing aspects on effective and non-effective implementation of technology-based counselling. This could be telephone-based, web-based or mobile-based interventions for people with dementia and informal carers. For the second primary objective, randomised controlled trials (RCTs) dealing with any outcomes will be included. Year of publication and language will not be restricted. We will search CINAHL, Cochrane Library, MEDLINE, PsycINFO and Web of Science up to April 2021. Additionally, we will perform web searching and citation tracking. To achieve the first primary objective, a Qualitative Comparative Analysis (QCA) will be conducted. The QCA enables us to identify necessary or sufficient components for a successful implementation. To reach the second primary objective, a meta-analysis will be performed with respect to potential clinical and statistical heterogeneity of RCTs. The revised Risk of Bias tool 2.0 will be used to check the risk of bias in RCTs. For all other study designs, the Mixed Method Appraisal Tool will be used.

Ethics and dissemination Ethics approval is not required for this review. We will disseminate our findings through scientific and non-scientific journal articles and conference presentations as well as formats directed to the public and decision-makers in healthcare. PROSPERO registration number CRD42021245473.

\section{Strengths and limitations of this study}

- One methodological strength of our review is the combined determination of effectiveness of technology-based counselling interventions and of successful implementation features of such interventions.

- A comprehensive literature search combining electronic key database search and supplementary search methods with no restrictions on publication date and outcomes is another methodological strength of our review.

- Qualitative Comparative Analysis might uncover effective and non-effective combination(s) of intervention components that might be seen as a methodological strength of our review.

- Stakeholder participation and involvement in the Qualitative Comparative Analysis, the determination of the outcomes of interest for the Grading of Recommendations, Assessment, Development and Evaluation (GRADE) approach as well as the interpretation of the review findings enables us to adopt the end-user perspectives and to strengthen the external validity of the review results.

- Since we expect only a few studies about technologybased counselling, this could result in limited data for the Qualitative Comparative Analysis.

\section{INTRODUCTION}

Description of the condition

Dementia is one of the major causes of disability and dependency among older people and is characterised by a progressive decline of cognitive functioning alongside other impairments, such as memory, executive functions, attention, emotional control and language. ${ }^{1}$ Nearly 50 million people have dementia worldwide, of whom approximately $60 \%$ live in low-income and middleincome countries. ${ }^{2}$ The number of new cases of dementia each year is estimated at over 
9.9 million worldwide. ${ }^{3}$ The average survival time after diagnosis seems to depend on the type of dementia; however, the length of survival is not related to age at diagnosis. ${ }^{4}$ Results of a meta-analysis demonstrate an average survival time of 4.11 years after the diagnosis of Lewy body dementia $(\mathrm{SD} \pm 4.10)$. People with a diagnosis of Alzheimer's disease have an average survival time of 5.66 years $(\mathrm{SD} \pm 5.32) .{ }^{5}$

Common consequences of dementia are unmet needs, which can cause behavioural and psychological symptoms. ${ }^{6}$ Hyperactivity and apathy show high persistence and incidence; depression and anxiety show low or moderate persistence and moderate incidence, and psychotic symptoms show low persistence and a moderate or low incidence. Around half of all the people with dementia are in need of assistance with personal care. According to an estimation by Prince $e t a l$, informal carers spent an average of 14 hours weekly supporting the person with dementia in activities of daily living (ADLs), such as dressing or bathing. Additionally, another 43 hours for instrumental activities of daily living (IADLs), for instance organisation of care or supervision, are needed. No significant differences between countries were identified. ${ }^{7}$

The majority of people with dementia live at home and receive care from a relative, mainly a spouse. ${ }^{8}$ Informal carers of people with dementia seem to be less healthy and might experience more difficulties and stress than informal carers of people with other chronic conditions. ${ }^{910}$ Three dimensions of burden for informal carers have been described: direct impact of caregiving on informal carers' life (eg, they lost control of their life or their health suffered), guilt (eg, they should do more or could do a better job), and frustration or embarrassment (eg, care recipient asks for too much help or carer is embarrassed or angered by the care recipient). ${ }^{11}$ Nevertheless, providing care does not have a purely negative connotation, because informal carers feel vindicated in their role or have developed coping strategies. ${ }^{12}$ Excessive informal carers' burden increases the risk of people with dementia having to move from their own home to a nursing home. ${ }^{13}$ The worldwide COVID-19 (SARS-CoV-2) pandemic raised great concerns for people with dementia that were related to a lack of information, difficulties in safeguarding procedures or social isolation. ${ }^{14}$ Supporting both people with dementia and their informal carers is essential in order to overcome anxiety, stress, agitation or withdrawal. ${ }^{15}$

\section{Description of the intervention}

Information technologies provide supportive options without the risk of infection. Beyond the current pandemic, the intensity of dementia care and the lack of available support services or existing barriers to get access-especially in rural areas-require additional and alternative support possibilities. ${ }^{16}$ Information technology can provide timely and individual support for people with dementia and informal carers. ${ }^{17-23}$ Various types of support are needed, such as information, education or counselling. ${ }^{24}$ Counselling is defined as a conversation therapy that involves a trained therapist listening to individuals and helping them to find ways to cope with an emotional issue. It is differentiated from information, education, instruction and case management. ${ }^{25} \mathrm{Few}$ studies have investigated the effects of counselling for people with dementia and their informal carers. ${ }^{26}$ Potential effects include changes in the attitudes of couples confronted with Alzheimer's disease towards becoming more acceptant of the diagnosis along with a more optimistic view for the future. ${ }^{27}$

\section{How the intervention might work}

Counselling can be provided through a face-to-face interaction, in a group, or through the use of technological channels, namely: the phone, email or live chat services. People who received telephone counselling believed that the intervention was attractive with regard to convenience, accessibility, control or inhibition. ${ }^{28} \mathrm{~A}$ recent mixed-methods study uncovered the needs and preferences of informal carers of people with dementia and shows that an online intervention might be an adequate format to provide individual support. From their perspective, tailored programmes that are simple to use and interaction with a professional within the online intervention are highly needed. ${ }^{29} \mathrm{~A}$ recent scoping review on virtual support groups for informal carers of people with dementia also highlights the need for tailored, easyaccessible and moderated online support. ${ }^{30}$ Therefore, online counselling might meet the needs of many clients and result in positive outcomes. ${ }^{31}$ For example, virtual appointments could reduce stress among carers because counselling sessions can be carried out from home. This is in particular of interest, if carers have a problem leaving their home where they care for a person with dementia. Online counselling can be differentiated from other online services (eg, web-based education or therapeutic interventions, therapeutic software or online activities, which are not stand-alone services). ${ }^{32}$

Alternatives to face-to-face interventions are crucial in the current COVID-19 pandemic and furthermore have the potential for a more timely and individualised support in dementia. Few systematic reviews investigated technology-based interventions for people with dementia and informal carers (eg, telephone counselling, ${ }^{33}$ or support interventions via computer and telephone. ${ }^{34}$ The review by Lins et al included nine randomised controlled trials (RCTs) and two qualitative studies. ${ }^{33}$ The mixedmethod review aimed to identify the efficacy of telephone counselling for informal carers of people with dementia and asked for the experiences of informal carers with the intervention. The results demonstrate moderate evidence that telephone counselling without other intervention components can reduce depressive symptoms in informal carers. The experiences of informal carers included barriers and facilitators for successful implementation, counsellor's emotional attitude and content of the telephone counselling. The authors concluded 
that the efficacy of the intervention was influenced by a lack of knowledge related to the aspects of telephone counselling. The review by Waller et al included studies related to interventions delivered by a computer $(n=10)$, multiple modalities $(n=9)$ or telephone $(n=15) \cdot{ }^{34}$ Due to the expanding number of related publications, one could assume an increasing demand for better support for informal carers of people with dementia. In conclusion, a need for support and advice for the person with dementia and the informal carer as a dyad was identified.

An overview of systematic reviews investigated psychosocial interventions in dementia and identified counselling and psychotherapy as the second largest category of interventions for informal carers, showing some evidence confirming the effectiveness of these interventions. ${ }^{35}$ Technology-based interventions were also effective, but components or aims of these interventions were not described. As in the case of the reviews by Lins $e t$ al and Waller et al, conditions of telephone-based counselling or technology-based interventions were not described in detail. ${ }^{33} 34$

An update and extension of the review by Lins $e t$ al is timely and warranted. ${ }^{33}$ In addition to telephone-based counselling, we will cover other technologies such as computer or web-based interventions. Our review itself will address both the target group of informal carers of people with dementia and studies that report on interventions for people with dementia themselves. That step would fulfil the global vision of evidence-based care in dementia, providing care and support for the needs of people with dementia and their informal carers to enhance their dignity, respect, autonomy and equality. ${ }^{36}$

\section{Objectives}

Although there is some evidence that people with dementia and informal carers might benefit from technology-based counselling, ${ }^{33} 34$ it remains unclear what features of those interventions relate to successful outcomes and what aspects of implementation are necessary and successful. Our review will identify conditions of a successful implementation of technology-based counselling interventions in dementia and investigate the effectiveness of those interventions. The results will present the effectiveness, that is, beneficial impact and potential adverse events of technology-based counselling for people with dementia and their carers. This is necessary to guide clinical practice concerning the use of such interventions. A comprehensive overview of successful and non-successful intervention components will facilitate a structured guidance for clinical implementation. The objectives of our review are therefore (1) to identify conditions that are aligned with successful implementation of technology-based counselling interventions in dementia (ie, implementation facilitators and barriers) and (2) to investigate the effectiveness of these interventions. The following two review questions are raised:

- What are necessary or sufficient conditions that are aligned with successful implementation and effective outcomes of technology-based counselling interventions for people with dementia and their carers?

- How effective are technology-based counselling interventions for people with dementia and their carers?

\section{METHODS AND ANALYSIS}

We will conduct a mixed-methods systematic review using (1) the methodological approach of Qualitative Comparative Analysis (QCA), aimed at identifying the features of or conditions for successful interventions ${ }^{37}$ and (2) a meta-analysis, aimed at determining the overall effectiveness of technology-based counselling in dementia. Preferred Reporting Items for Systematic Review and Meta-analysis Protocols (PRISMA-P) was used to structure this systematic review protocol. ${ }^{38}$

\section{Eligibility criteria}

To reach our review objectives, we are interested in all information concerning the development, evaluation and implementation of technology-based counselling interventions in dementia. Therefore, we defined the following eligibility criteria.

\section{Populations}

We will include studies on interventions for people with dementia and/or their informal carers. There will be no restrictions concerning setting, gender, age, ethnic background, type and severity of dementia, or relationship between people with dementia and their informal carers. Studies on people with mild cognitive impairment or their informal carers will be excluded. Studies with a mixed sample of informal carers of people with dementia and other conditions will be included if the results for the dementia subgroup have been reported separately. We will also exclude studies on interventions for health and social care professionals.

\section{Interventions}

We will include tailored technology-based counselling interventions for people with dementia and/or their informal carers. Technology-based interventions use technologies such as telephone, computer and web-based or mobile-based applications to enable the delivery of the intervention instantly and/or remotely. Counselling is a complex intervention that might consist of different intervention components or be part of multicomponent interventions. To assess the impact of counselling, we will focus on interventions with a core component of counselling. Thus, we will include tailored counselling interventions provided by health and social care professionals such as nurses, physicians or psychologists aiming to support people with dementia and/or their informal carers (eg, focusing management of daily living, disease and care management as well as functioning and participation). Therefore, the interventions of interest will be technology-based, provided human-to-human, but may be asynchronous. Studies with interventions that might use counselling as a component of complex care programmes and mainly focus on coordination of care 
such as case management will be excluded. We will also exclude specific and standardised interventions such as cognitive-behavioural therapy, genetic counselling as well as counselling with regard to predictive diagnostics, screening for dementia or diagnostic procedures. Counselling interventions provided by non-professionals (ie, non-paid) will be excluded.

\section{Comparators}

Technology-based counselling will be compared with no treatment, to standard or usual care, and to in presence or face-to-face counselling. Comparisons between various technology-based interventions will also be considered for inclusion.

\section{Outcomes}

We will include all features of effective and non-effective intervention implementation and there will be no restrictions regarding outcomes on the effectiveness of interventions. Our primary outcome is defined as the impact of technology-based counselling on depressive symptoms experienced by people with dementia and their carers. As secondary outcomes, we will consider outcomes on the (1) patient and carer levels (such as burden, well-being, delay or avoidance of transition to a nursing home, decision making and acceptance of as well as satisfaction with technology-based counselling) and on the (2) healthcare system level (such as usage and costs of technology-based counselling). ${ }^{34} 35$ We do not expect a negative impact from counselling interventions for people with dementia and carers. However, adverse events will be included.

\section{Design of primary studies}

The first primary objective requires evidence from various study designs addressing aspects of implementation success such as qualitative studies, descriptive quantitative studies (eg, before-after studies, clinical controlled studies, cross-sectional studies, surveys) and mixedmethods research.

To meet the second primary objective, we will include individual and cluster parallel-group RCTs, also RCTs using cross-over and stepped-wedge designs.

\section{Information sources}

We will perform a comprehensive literature search combining an electronic database search with supplementary search methods and with no limitations regarding language and year of publication. ${ }^{39} 40$

\section{Electronic database search}

We will search CINAHL, Cochrane Library including Cochrane Central Register of Controlled Trials (CENTRAL), MEDLINE via PubMed, PsycINFO via Ovid and the Web of Science Core Collection up to April 2021 with no use of filters and limiters.

\section{Supplementary search methods}

Google Scholar will be used for free web searching with highly relevant free text terms in order to identify further studies and conference abstracts not yet identified as well as grey literature, thus minimising publication bias. We will search further primary studies using (1) hand searching of revealed reviews and (2) forward and backward citation tracking of included studies using Scopus. To identify ongoing studies, we will search the International Clinical Trials Registry Platform (ICTRP) via CENTRAL, a meta study registry provided by the WHO covering national registries from 17 countries worldwide.

\section{Search update}

An update for potentially relevant studies will be conducted in electronic databases 1 year after the initial search. For the search update, we will use the same search methods and strategies.

\section{Search strategy}

We will use database-specific search strategies based on database-specific controlled vocabulary, for instance, MeSH (MEDLINE) and unspecified free-text terms. The search strategies will be developed and peer-reviewed within the review group using Peer Review of Electronic Search Strategies (PRESS). A researcher experienced in designing search strategies will support us during that process. ${ }^{41}$ Our search strategy will contain three components (Dementia, Technology and Counselling). Search terms and their synonyms will be identified using an earlier study by our review group, ${ }^{33}$ an orienting search using MEDLINE via PubMed and through the experience of the review group members. Additionally, text mining of titles and abstracts of previously known studies will be applied to identify relevant text terms used in the core literature. Based on the identified search terms, we will look for further search terms with the help of a thesaurus ${ }^{42}$ and by checking entry terms given in the Medical Subject Headings (MeSH) browser. For unspecified free text terms, we will search title, abstract, and keyword fields, if available. Controlled vocabulary will be used in the corresponding fields and additionally in the title, abstract and keyword search fields in order to cover publications not yet tagged with controlled vocabulary. ${ }^{43}$ The final search strategy per database is provided in online supplemental file 1.

\section{Study records}

Two reviewers will independently screen the identified titles, abstracts, and, if eligible, full texts for inclusion and exclusion using the Rayyan web app. ${ }^{44}$ We will discuss conflicting results within the screening team and with a third member of the review group-if necessary. Two reviewers will develop and pilot a data extraction sheet using ten percent of the included studies. Subsequently, one reviewer will extract data. A second reviewer will check the data extraction for its accuracy. We will discuss conflicting results within the data extracting team and, if necessary, also with a third member of the review group. 


\section{Data items}

We will extract data on study characteristics (eg, study design, country), participants' characteristics (eg, age, gender, type and severity of dementia), intervention and control group characteristics (eg, type, format, length, frequency), outcome(s) or phenomena of interest (eg, implementation facilitators and barriers), measurement(s) and results. In addition, we will extract data according to the criteria of the Template for Intervention Description and Replication (TIDieR) checklist and the updated Criteria for Reporting the Development and Evaluation of Complex Interventions (CReDECI-2) guideline to provide a description of the reported intervention characteristics and their theoretical background. ${ }^{4546}$

\section{Outcomes and prioritisation}

We will involve our advisory board in determining the outcomes of interest following the Grading of Recommendations, Assessment, Development and Evaluation (GRADE) approach (for details see below). ${ }^{47}$

\section{Risk of bias and critical appraisal in individual studies}

We will assess the risk of bias in RCTs using the revised risk of bias tool (RoB 2.0). ${ }^{48}$ For all other study designs, we will use the Mixed Method Appraisal Tool, a comprehensive critical appraisal tool provided by Hong et al. ${ }^{49}$ All appraisals will be conducted by two independent reviewers. We will discuss conflicting results within the appraisal team and, if necessary, with a third member of the review group.

\section{Data synthesis}

Qualitative Comparative Analysis

To meet our first primary objective, we will conduct a QCA to identify conditions of interventions that are related to successful implementation and effective outcomes. Key elements of the QCA are the configurations of the conditions. These are different scenarios of intervention components and potential facilitators as well as barriers of their implementation. The QCA enables us to identify necessary or sufficient components for implementation aspects and successful interventions. QCA contains the steps which are displayed in the following subchapters. ${ }^{37}$

\section{Building the data table using selected conditions and outcomes}

Unlike systematic reviews on effectiveness interventions, the QCA approach considers various study designs. Studies ('cases' in the QCA terminology) will be added or removed in an iterative process until a later stage of the research.

The selection and definition of the conditions and outcomes is based on the preliminary findings from the literature. ${ }^{50}$ The conditions will be grouped into different domains such as setting and participants' characteristics, intervention characteristics or planned and unplanned measures to facilitate implementation. To structure our conditions and domains, we will develop a logic model. The number of conditions is expected to be relatively low, and for each condition a clear hypothesis will be formulated according to its expected relation to the outcome. ${ }^{37}$ Identified conditions and outcomes will be mapped in a raw data table and calibrated towards their so-called set membership. To prepare the calibration process, a coding framework is needed to provide instructions and values for the data extraction. For each case (=included study), the conditions and outcomes will be determined in crisp-sets with dichotomous values of 0 or 1 , or with fuzzy-sets for degrees of membership, transformed in values between 0 and 1 .

\section{Constructing a truth table and resolving contradictory configurations}

In a next step, the possible combinations of conditions will be tabulated. Each line in the table represents a configuration of possible conditions using logical operators. The number of configurations will be $2 * \mathrm{k}$, where ' $\mathrm{k}$ ' represents the number of conditions. The configuration represents a potential set where studies can be members or non-members. Studies showing the same configuration will be included in one set and studies showing different configurations will be included in other sets. Therefore, so-called truth tables using the dichotomous or transformed values of crisp sets and fuzzy sets will be developed when configurations (1) are supported by (one or more) cases, (2) are counter-intuitive or showed an identical combination of conditions, (3) occurred infrequently and (4) triggered positive and negative outcomes at the same time.

Boolean minimisation and consideration of the 'logical remainders' cases

We will subsequently use Boolean minimisation to identify configurations that are observed to adequately lead to a sufficient and specific outcome. We will then consider the plausibility of the configurations and search for consistent conclusions in individual studies (consideration of logical remainders cases). Identified configurations that are sufficiently specific can form different paths towards an outcome. ${ }^{50}$ Therefore, coefficients of coverage will be expressed, and the theoretical relevance will be considered. Furthermore, a 'return to the cases' step will help us to evaluate how these results are represented in a study and across studies. ${ }^{4751}$

\section{Interpretation of the results}

The interpreted QCA results will be used for the development of a logical model that can support the practice of technology-based counselling. Furthermore, the newly introduced logical model has the potential to sufficiently inform further research activities in order to compromise inadequate knowledge about the effective steps of implementation. We will use an appropriate software for that analytic step.

\section{Meta-analysis}

To meet our second primary objective, we will perform a meta-analysis with respect to the potential clinical and statistical heterogeneity of RCTs. Clinical heterogeneity 
will be indicated by the comparability of interventions based on intervention characteristics and components. ${ }^{52}$ Groupings consisting of studies with similar characteristics and interventions might reach comparability. Therefore, it will be possible to conduct stratified meta-analyses given a sufficient number of studies across study groups. We will evaluate the statistical heterogeneity using the $\mathrm{I}^{2}$ method. ${ }^{53}$ A value higher than $50 \%$ will be regarded as significantly heterogeneous. Here, we will use the randomeffects model and in the case of non-significant heterogeneity, we will use the fixed-effects model to calculate the pooled effect sizes. ${ }^{54} \mathrm{~A} p<0.05$ will be considered as statistically significant. For meta-analysis of dichotomous results, we will calculate the relative risks (RR) with $95 \%$ CI. For the meta-analysis of continuous results, we will use the mean difference (MD) and 95\% CI. If different outcome measurements have been used, we will use the standardised mean difference (SMD) and 95\% CI. To assess the influence of different conditions on the effects of technology-based counselling (eg, setting, recipient, facilitator and type of the intervention), we will conduct sensitivity analyses based on subgroup meta-analyses. ${ }^{55}$ We will use the software ' $R$ ' in its current version for our calculations. The results of the meta-analysis will be presented in forest plots. ${ }^{56}$

If the previously described assessments indicate heterogeneous interventions and study results, we will provide a narrative synthesis based on tabular data extraction. Additionally, we will summarise effects, risk of bias, intervention and study characteristics graphically by means of a Harvest plot to report appropriately on the complexity and heterogeneity of the included studies. ${ }^{5758}$

\section{Meta-bias}

Given a sufficient number of included studies to meet our second primary objective, risk for publication bias will be assessed graphically using a funnel plot complemented by a statistical test. ${ }^{59}$

\section{Confidence in cumulative evidence}

The quality of evidence will be assessed using the GRADE approach by rating the quality of evidence as high, moderate, low or very low. ${ }^{60}$ The combined results of meta-analyses and the GRADE approach will be presented in a summary of findings table. ${ }^{61}$

\section{Patient and public involvement}

We will include representatives of people with dementia and informal carers, that is, people engaged in a support organisation that supports dementia care, and an informal carer of a person with dementia. The advisory board will consist of two or three people. Recommendations by the advisory board will be used for the qualitative steps of the QCA, where experience and knowledge of the subject are needed for the identification and the assessment of conditions that are important for the intervention. Furthermore, the advisory board will be involved in determining the outcomes of interest for the GRADE approach as well as the interpretation of the review findings. ${ }^{47}$

\section{Ethics and dissemination}

Ethics approval is not required for this review protocol. The members of the advisory board participate voluntarily in the review process. Ongoing informed consent will be obtained.

Our dissemination strategy uses multiple channels of distribution to share our review work and results with academic and non-academic stakeholders, the society and interested individuals. ${ }^{62}$

The final review will be published in an international open access journal relevant in the field of dementia care. Additionally, we will discuss our review results with experts at national and international conferences. Plain language summaries of the review will be placed on our institutional websites and social media profiles (eg, Twitter, ResearchGate). A second review report will be published in a non-academic journal to share our review results with other interested groups such as practitioners and carers of people with dementia. Furthermore, we will participate in interactive mass media platforms to enable interested groups to ask questions. A cartoon video will be accessible and disseminated online. Additionally, the results will be printed in newsletters of organisations representing people with dementia as well as patient advocacy groups such as Deutsche Alzheimer Gesellschaft, Alzheimer Schweiz, Österreichische Alzheimer Gesellschaft and Alzheimer Europe. Furthermore, we will contact insurance companies and other stakeholders to disseminate our research results.

\section{Author affiliations}

${ }^{1}$ Institute of Health and Nursing Science, Medical Faculty, Martin Luther University Halle-Wittenberg, Halle (Saale), Germany

${ }^{2}$ Center for Dementia Care, Institute of Applied Nursing Sciences, Department of Health, Eastern Switzerland University of Applied Sciences, St Gallen, Switzerland ${ }^{3}$ Department of Clinical Research, University Hospital Basel, University of Basel, Basel, Switzerland

Acknowledgements We thank Thomas Nordhausen from the Institute for Health and Nursing Science at Martin Luther University Halle-Wittenberg for peerreviewing and critically appraising our search strategies.

Contributors Substantial contributions to the conception or design of the work: $\mathrm{JH}, \mathrm{GL}, \mathrm{GM}$ and $\mathrm{AB}$. Drafting the work: $\mathrm{JH}$ and $\mathrm{AB}$. Revising it critically for important intellectual content: JH, GL, FW, DB, GM and AB. Final approval of the version to be published: JH, GL, FW, DB, GM and AB. Agreement to be accountable for all aspects of the work in ensuring that questions related to the accuracy and integrity of any part of the work are appropriately investigated and resolved: JH, GL, FW, DB, GM and $A B$.

Funding This study is funded by the German Federal Ministry of Education and Research (BMBF), grant number (01KG2110).

Disclaimer The funder has no influence on the design, conduct, results of this research nor its preparation and submission for publication.

Competing interests None declared.

Patient consent for publication Not applicable.

Provenance and peer review Not commissioned; externally peer reviewed.

Supplemental material This content has been supplied by the author(s). It has not been vetted by BMJ Publishing Group Limited (BMJ) and may not have been peer-reviewed. Any opinions or recommendations discussed are solely those 
of the author(s) and are not endorsed by BMJ. BMJ disclaims all liability and responsibility arising from any reliance placed on the content. Where the content includes any translated material, BMJ does not warrant the accuracy and reliability of the translations (including but not limited to local regulations, clinical guidelines, terminology, drug names and drug dosages), and is not responsible for any error and/or omissions arising from translation and adaptation or otherwise.

Open access This is an open access article distributed in accordance with the Creative Commons Attribution Non Commercial (CC BY-NC 4.0) license, which permits others to distribute, remix, adapt, build upon this work non-commercially, and license their derivative works on different terms, provided the original work is properly cited, appropriate credit is given, any changes made indicated, and the use is non-commercial. See: http://creativecommons.org/licenses/by-nc/4.0/.

\section{ORCID iDs}

Julian Hirt http://orcid.org/0000-0001-6589-3936

Anja Bieber http://orcid.org/0000-0002-9506-2532

\section{REFERENCES}

1 WHO. Icd-11 for mortality and morbidity statistics. neurocognitive disorders, 2019. Available: https://www.who.int/classifications/icd/ en/

2 WHO. Dementia, 2019. Available: https://www.who.int/news-room/ fact-sheets/detail/dementia

3 Prince M, Wimo A, Guerchet M. World Alzheimer report 2015: the global impact of dementia: an analysis of prevalence, incidence, cost and trend London, 2015. Available: https://www.alz.co.uk/research/ WorldAlzheimerReport2015.pdf [Accessed 19 Mar 2020].

4 Brodaty H, Seeher K, Gibson L. Dementia time to death: a systematic literature review on survival time and years of life lost in people with dementia. Int Psychogeriatr 2012;24:1034-45.

5 Mueller C, Soysal P, Rongve A, et al. Survival time and differences between dementia with Lewy bodies and Alzheimer's disease following diagnosis: a meta-analysis of longitudinal studies. Ageing Res Rev 2019;50:72-80.

6 Hung L, Lee PA, Au-Yeung AT, et al. Adopting a clinical assessment framework in older adult mental health. J Psychosoc Nurs Ment Health Serv 2016;54:26-31.

7 Prince M, Prina M, Guerchet M. World Alzheimer report 2013: journey of caring: an analysis of long-term care for dementia London, 2013. Available: https://www.alz.co.uk/research/WorldAlzheimerRe port2013.pdf [Accessed 19 Mar 2020].

8 Wimo A, Gauthier S, Prince M. Global estimates of informal care, 2018. Available: https://www.alz.co.uk/adi/pdf/global-estimates-ofinformal-care.pdf [Accessed 15 May 2020].

9 Schoenmakers B, Buntinx F, Delepeleire J. Factors determining the impact of care-giving on caregivers of elderly patients with dementia. A systematic literature review. Maturitas 2010;66:191-200.

10 Isik AT, Soysal P, Solmi M, et al. Bidirectional relationship between caregiver burden and neuropsychiatric symptoms in patients with Alzheimer's disease: a narrative review. Int J Geriatr Psychiatry 2019;34:1326-34.

11 Springate BA, Tremont G. Dimensions of caregiver burden in dementia: impact of demographic, mood, and care recipient variables. Am J Geriatr Psychiatry 2014;22:294-300.

12 Lloyd J, Patterson T, Muers J. The positive aspects of caregiving in dementia: a critical review of the qualitative literature. Dementia 2016;15:1534-61.

13 Cepoiu-Martin M, Tam-Tham H, Patten S, et al. Predictors of longterm care placement in persons with dementia: a systematic review and meta-analysis. Int J Geriatr Psychiatry 2016;31:1151-71.

14 Wang H, Li T, Barbarino P, et al. Dementia care during COVID-19. The Lancet 2020;395:1190-1.

15 WHO. ADI offers advice and support during COVID-19, 2020. Available: https://www.alz.co.uk/news/adi-offers-advice-andsupport-during-covid-19 [Accessed 05 May 2020]

16 Innes A, Morgan D, Kosteniuk J, et al. Dementia care in rural and remote settings: a systematic review of informal/family caregiving. Maturitas 2011;68:34-46.

17 Bejan A, Gündogdu R, Butz K, et al. Using multimedia information and communication technology (ICT) to provide added value to reminiscence therapy for people with dementia : Lessons learned from three field studies. Z Gerontol Geriatr 2018;51:9-15.

18 D'Onofrio G, Sancarlo D, Ricciardi F, et al. Information and communication technologies for the activities of daily living in older patients with dementia: a systematic review. J Alzheimers Dis 2017;57:927-35.
19 Lauriks S, Reinersmann A, Van der Roest HG, et al. Review of ICTbased services for identified unmet needs in people with dementia. Ageing Res Rev 2007;6:223-46.

20 Lucero RJ, Fehlberg EA, Patel AGM, et al. The effects of information and communication technologies on informal caregivers of persons living with dementia: a systematic review. Alzheimers Dement 2019;5:1-12.

21 McHugh JE, Wherton JP, Prendergast DK, et al. Identifying opportunities for supporting caregivers of persons with dementia through information and communication technology. Gerontechnology 2013;10

22 Noone C, McSharry J, Smalle M, et al. Video calls for reducing social isolation and loneliness in older people: a rapid review. Cochrane Database Syst Rev 2020;5:CD013632.

23 Pleasant M, Molinari V, Dobbs D, et al. Effectiveness of online dementia caregivers training programs: a systematic review. Geriatr Nurs 2020;41:921-35

24 Whitlatch CJ, Orsulic-Jeras S. Meeting the informational, educational, and psychosocial support needs of persons living with dementia and their family caregivers. Gerontologist 2018;58:S58-73

25 NHS. Counselling. Available: https://www.nhs.uk/conditions/ counselling/2017 [Accessed 17Jan 2020].

26 Dickinson C, Dow J, Gibson G, et al. Psychosocial intervention for carers of people with dementia: what components are most effective and when? A systematic review of systematic reviews. Int Psychogeriatr 2017;29:31-43.

27 Elvish R, Lever S-J, Johnstone J, et al. Psychological interventions for carers of people with dementia: a systematic review of quantitative and qualitative evidence. Couns Psychother Res 2013;13:106-25.

28 Reese RJ, Conoley CW, Brossart DF. The attractiveness of telephone counseling: an empirical investigation of client perceptions. J Couns Dev 2006;84:54-60.

29 Teles S, Paúl C, Sosa Napolskij M, et al. Dementia caregivers training needs and preferences for online interventions: a mixed-methods study. J Clin Nurs 2020. doi:10.1111/jocn.15445. [Epub ahead of print: 05 Aug 2020].

30 Armstrong MJ, Alliance S. Virtual support groups for informal caregivers of individuals with dementia: a scoping review. Alzheimer Dis Assoc Disord 2019;33:362-9.

31 Harris B, Birnbaum R. Ethical and legal implications on the use of technology in counselling. Clin Soc Work J 2015;43:133-41.

32 Dowling M, Rickwood D. Online counseling and therapy for mental health problems: a systematic review of individual synchronous interventions using chat. J Technol Hum Serv 2013;31:1-21.

33 Lins S, Hayder-Beichel D, Rücker G, et al. Efficacy and experiences of telephone counselling for informal carers of people with dementia. Cochrane Database Syst Rev 2014;9:CD009126.

34 Waller A, Dilworth S, Mansfield E, et al. Computer and telephone delivered interventions to support caregivers of people with dementia: a systematic review of research output and quality. BMC Geriatr 2017;17:265.

35 Clarkson P, Hughes J, Xie C, et al. Overview of systematic reviews: effective home support in dementia care, components and impactsStage 1, psychosocial interventions for dementia. $J$ Adv Nurs 2017;73:2845-63.

36 WHO. Global action plan on the public health response to dementia 2017 - 2025, 2017. Available: https://apps.who.int/iris/bitstream/ handle/10665/259615/9789241513487-eng.pdf?sequence $=1$

37 Rihoux B, Ragin CC, eds. Configurational comparative methods: Qualitative comparative analysis (QCA) and related techniques. Los Angeles: SAGE, 2009.

38 Moher D, Shamseer L, Clarke M, et al. Preferred reporting items for systematic review and meta-analysis protocols (PRISMA-P) 2015 statement. Syst Rev 2015;4:1.

39 Cooper C, Booth A, Varley-Campbell J, et al. Defining the process to literature searching in systematic reviews: a literature review of guidance and supporting studies. BMC Med Res Methodol 2018;18:85

40 Lefebvre C, Glanville J, Briscoe S. Searching for and selecting studies. In: Higgins JPT, Thomas J, eds. Cochrane Handbook for systematic reviews of interventions. 2 edn. Hoboken: Wiley Online Library, 2019: 67-108.

41 McGowan J, Sampson M, Salzwedel DM, et al. PRESS peer review of electronic search strategies: 2015 Guideline Statement. J Clin Epidemiol 2016;75:40-6.

42 Dictionary.com. Thesaurus.com, 2020. Available: http://www. thesaurus.com/[Accessed 28.04.2020].

43 Rodriguez RW. Comparison of indexing times among articles from medical, nursing, and pharmacy journals. Am J Health Syst Pharm 2016;73:569-75. 
44 Ouzzani M, Hammady H, Fedorowicz Z, et al. Rayyan-a web and mobile app for systematic reviews. Syst Rev 2016;5:210.

45 Hoffmann TC, Glasziou PP, Boutron I, et al. Better reporting of interventions: template for intervention description and replication (TIDieR) checklist and guide. BMJ 2014;348:g1687.

46 Möhler R, Köpke S, Meyer G. Criteria for reporting the development and evaluation of complex interventions in healthcare: revised guideline (CReDECI 2). Trials 2015;16:204.

47 Langer G, Meerpohl JJ, Perleth M, et al. GRADE-Leitlinien: 2. Formulierung Der Fragestellung und Entscheidung über wichtige Endpunkte. Zeitschrift für Evidenz, Fortbildung und Qualität im Gesundheitswesen 2012;106:369-76.

48 Sterne JAC, Savović J, Page MJ, et al. Rob 2: a revised tool for assessing risk of bias in randomised trials. BMJ 2019;366:14898.

49 Hong QN, Fàbregues S, Bartlett G, et al. The mixed methods appraisal tool (MMAT) version 2018 for information professionals and researchers. Education for Information 2018;34:285-91.

50 Schneider CQ, Wagemann C. Standards of good practice in qualitative comparative analysis (QCA) and Fuzzy-Sets. Comp Sociol 2010;9:397-418.

51 Kahwati L, Jacobs S, Kane H, et al. Using qualitative comparative analysis in a systematic review of a complex intervention. Syst Rev 2016;5:82.

52 West SL, Gartlehner G, Mansfield AJ. Comparative effectiveness review methods: clinical heterogeneity. agency for. Rockville: Healthcare Research and Quality, 2010.
53 Higgins JPT, Thompson SG, Deeks JJ, et al. Measuring inconsistency in meta-analyses. BMJ 2003;327:557-60.

54 Hedges LV, Vevea JL. Fixed- and random-effects models in metaanalysis. Psychol Methods 1998;3:486-504.

55 Khan KS, Kunz R, Kleijnen J. Systematic reviews to support evidence-based medicine. Hodder \& Stoughton, 2011

56 Lewis S, Clarke M. Forest plots: trying to see the wood and the trees. BMJ 2001;322:1479-80.

57 Higgins JPT, López-López JA, Becker BJ, et al. Synthesising quantitative evidence in systematic reviews of complex health interventions. BMJ Glob Health 2019;4:e000858.

58 Ogilvie D, Fayter D, Petticrew M, et al. The harvest plot: a method for synthesising evidence about the differential effects of interventions. BMC Med Res Methodol 2008;8:1-8.

59 Egger M, Davey Smith G, Schneider M, et al. Bias in meta-analysis detected by a simple, graphical test. BMJ 1997;315:629-34.

60 Schünemann HB, Guyatt Jan;, Gordon; Oxman A. Grade handbook: handbook for grading the quality of evidence and the strength of recommendations using the grade approach, 2013.

61 Guyatt GH, Thorlund K, Oxman AD, et al. Grade guidelines: 13. preparing summary of findings tables and evidence profilescontinuous outcomes. J Clin Epidemiol 2013;66:173-83.

62 Cochrane. Checklist and guidance: for disseminating findings from Cochrane intervention reviews: version 1.0. project in collaboration with Cochrane knowledge translation and Cochrane Norway, 2019. Available: https://training.cochrane.org/sites/training.cochrane.org/ files/public/uploads/Checklist\%20FINAL\%20version\%201.0.pdf 